\title{
A New Method for Bilateral Teleoperation Passivity under Varying Time Delays
}

\author{
Farzad Hashemzadeh, ${ }^{1,2}$ Iraj Hassanzadeh, 1,2 \\ Mahdi Tavakoli, ${ }^{2}$ and Ghasem Alizadeh ${ }^{1}$ \\ ${ }^{1}$ Control Engineering Department, Faculty of Electrical and Computer Engineering, \\ University of Tabriz, Tabriz 51664, Iran \\ ${ }^{2}$ Department of Electrical and Computer Engineering, University of Alberta, Edmonton, \\ $A B$, Canada T6G 2 V4
}

Correspondence should be addressed to Farzad Hashemzadeh, farzad.hashemzadeh@ualberta.ca

Received 23 December 2011; Revised 5 April 2012; Accepted 20 April 2012

Academic Editor: Alexander Pogromsky

Copyright (C) 2012 Farzad Hashemzadeh et al. This is an open access article distributed under the Creative Commons Attribution License, which permits unrestricted use, distribution, and reproduction in any medium, provided the original work is properly cited.

\begin{abstract}
A new framework is proposed to mitigate the adverse effect of time-varying time delays on the passivity of a teleoperation system. To this end, the communication channel with time-varying delays is modeled as a constant-delay channel along with additive output disturbances. Then, disturbance estimator blocks are added in each of the feedforward and feedback paths to estimate these disturbances and to compensate for them. In the disturbance estimator block, there is a need for a virtual time-varying delay block such that the overall communication channel can be seen as one with a constant delay. We also propose a method for determining this virtual delay. Two PHANToM haptic devices connected through a communication channel with time-varying delays are considered for a case study. Simulation and experimental results confirm the efficiency of the proposed method in terms of passivating the teleoperation system in the presence of time-varying delays.
\end{abstract}

\section{Introduction}

Using a telerobotic system, a human operator can carry out tasks in a remote environment. Different applications of telerobotic systems vary from telesurgery to space manipulation. In telerobotics applications with a distance between local and remote robots, there will be a time delay in the communication channel of the system. The time delay in the closed-loop system can destabilize the telerobotic systems [1-3]. Control schemes have been developed to compensate for the time delay, most of which are based on the passivity theory. Passivitybased control schemes [4-9] are inspired from energy interaction between interconnected systems [10]. To this end, Anderson and Spong [4] proposed scattering schemes based on the passivity theory. Another prominent passivity-based scheme is the wave variable formulation 
for a two-port network proposed by Neimeyer and Slotine [5, 6]. These passivity-based approaches can guarantee the passivity of bilateral teleoperation systems just for constant time delay and cannot preserve the passivity for varying time delays [11]. Another important framework in stability analysis of teleoperation systems is based on input-to-state stability and small-gain conditions [12-14].

In the passivity analysis of telerobotics systems using wave variable, wave signals are encoded and decoded before and after transmission based on the power signals (force and velocity) in such a way that the communication channel remains passive. The scattering and the wave variable approaches are the best-known methods in the passivity approach and have been the subject of recent studies concerning teleoperation under varying delays. An extension of the scattering approach to the case of varying time delays is reported in [7], in which a small positive gain is added in communication channel to dissipate the extra energy generated due to the distorted scattered signals caused by varying time delay. The gain should be less than $1-\dot{T}$, where $T$ is the instantaneous value of the varying time delay such that communication channel remains passive. Also, an extended version of the wave variable approach with varying time delay was reported in [15] in which to have the passive channel, the derivative of the time delay should be less than one.

Considering the effect of varying time delay on the stability and transparency of the network, in this paper a method is developed to improve the stability and transparency of the resultant network which is based on modeling the variable time delay with constant time delay, and additive disturbance. The simulation and experiment results on the tracking performance and passivity measurement of the varying delayed network show the efficiency of the proposed method to the typical wave variable scheme.

In the later part of the paper passivity and wave variable background is studied in Section 2, and passivity of the channel with respect to time delay is studied in Section 3. Main contribution is studied in Section 4. In Section 5 and 6 simulation and experiment results of the proposed method on two PHANToM haptic devices show the efficiency of the proposed method, and conclusion of the paper is considered in Section 7.

\section{Passivity and Wave Variable Background}

To study the stability of the teleoperation system, the network model could be considered based on the idea of passivity. The passivity represents mathematical description of the physical concepts of power and energy.

The passivity can be formally defined as follows.

Definition 2.1. A two-port network, Figure 1, is said to be passive if the total energy delivered to the network, $(t)=\int_{-\infty}^{t}\left(\dot{x}_{m}(\tau)^{T} f_{m}(\tau)-\dot{x}_{s}(\tau)^{T} f_{s}(\tau)\right) d \tau$, at its input and output ports is positive. In the case of master-slave telerobotics, if it is assumed that the operator and the environment are passive systems, then the sufficient condition of stability is that the teleoperator channel must be passive [16, 17]. The passivity of traditional teleoperator systems is very sensitive to transmission delays which can destroy passivity of the entire system by altering the power flow in the system and generating excess energy. To study stability in the presence of varying time delay the wave variable transformations [18] and passivity concepts [19] are used in this study. First, let us define the power delivered to the communication system as

$$
P_{\text {in }}(\tau)=\dot{x}_{m}(\tau)^{T} f_{m}(\tau)-\dot{x}_{s}(\tau)^{T} f_{s}(\tau),
$$




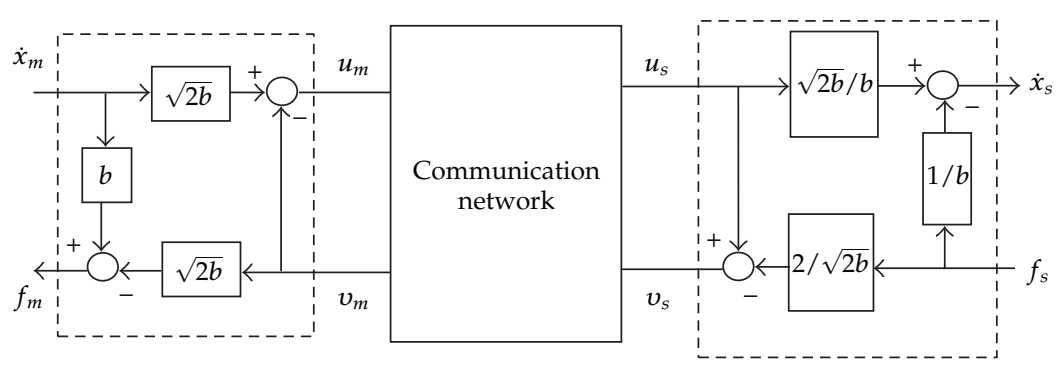

Figure 1: Wave variable with communication channel.

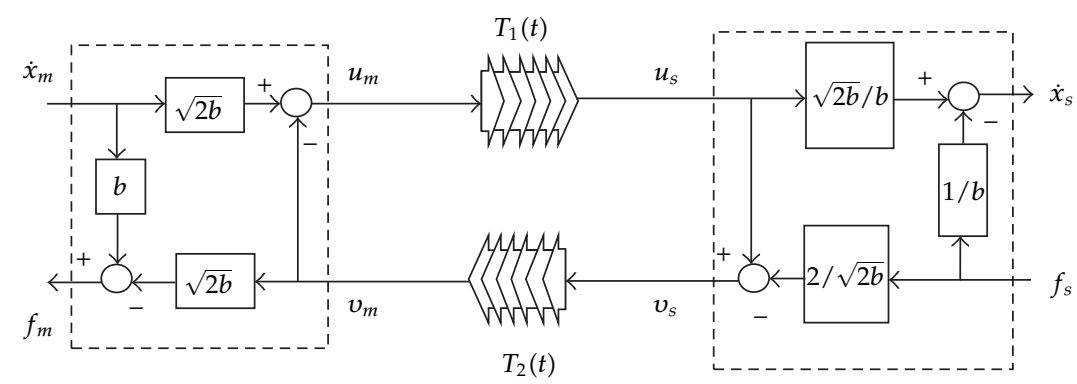

Figure 2: Conventional wave variable scheme with varying time delay.

where $f_{m}$ and $\dot{x}_{m}$ and $f_{s}$ and $\dot{x}_{m}$ are master and slave forces and velocities, respectively. The wave variables are defined as follows:

$$
\begin{array}{ll}
u_{m}=\frac{1}{\sqrt{2 b}}\left(b \dot{x}_{m}+f_{m}\right), & v_{m}=\frac{1}{\sqrt{2 b}}\left(b \dot{x}_{m}-f_{m}\right), \\
u_{s}=\frac{1}{\sqrt{2 b}}\left(b \dot{x}_{s}+f_{s}\right), & v_{s}=\frac{1}{\sqrt{2 b}}\left(b \dot{x}_{s}-f_{s}\right) .
\end{array}
$$

After some simplifications the power transfer can also be rewritten as follows:

$$
P_{\text {in }}(\tau)=\frac{1}{2}\left(u_{m}(\tau)^{T} u_{m}(\tau)-v_{m}(\tau)^{T} v_{m}(\tau)-u_{s}(\tau)^{T} u_{s}(\tau)+v_{S}(\tau)^{T} v_{s}(\tau)\right)
$$

\section{Passivity of the Channel with respect to Time Delay}

One of the advantages of wave formalism is that passivity could be preserved in the presence of time delays. In the ideal case where there are no delays between the master and the slave the communication channel is clearly passive. However, a communication device introduces some transmission delays which are time-varying in general. 
The conventional wave variable scheme [20] is shown in Figure 2, where $u_{s}(t)=u_{m}(t-$ $\left.T_{1}(t)\right), v_{m}(t)=v_{s}\left(t-T_{2}(t)\right)$. The input-output relationships for each wave block are same as (2.2). The dissipated energy in the communication channel is given by

$$
\begin{aligned}
\int_{0}^{t} P(\tau) d \tau= & \int_{0}^{t}\left(\dot{x}_{m}^{T}(\tau) f_{m}(\tau)-\dot{x}_{s}^{T}(\tau) f_{s}(\tau)\right) d \tau \\
= & \frac{1}{2} \int_{0}^{t}\left(u_{m}^{T}(\tau) u_{m}(\tau)-u_{s}^{T}(\tau) u_{s}(\tau)+v_{s}^{T}(\tau) v_{s}(\tau)-v_{m}^{T}(\tau) v_{m}(\tau)\right) d \tau \\
= & \frac{1}{2} \int_{0}^{t}\left(u_{m}^{T}(\tau) u_{m}(\tau)\right) d \tau-\frac{1}{2} \int_{0}^{t}\left(u_{m}^{T}\left(\tau-T_{1}(\tau)\right) u_{m}\left(\tau-T_{1}(\tau)\right)\right) d \tau \\
& +\frac{1}{2} \int_{0}^{t}\left(v_{s}^{T}(\tau) v_{s}(\tau)\right) d \tau-\frac{1}{2} \int_{0}^{t}\left(v_{s}^{T}\left(\tau-T_{2}(\tau)\right) v_{s}\left(\tau-T_{2}(\tau)\right)\right) d \tau
\end{aligned}
$$

More simplification gives

$$
\begin{aligned}
\int_{0}^{t} P(\tau) d \tau= & \frac{1}{2} \int_{0}^{t}\left(u_{m}^{T}(\tau) u_{m}(\tau)\right) d \tau-\frac{1}{2} \int_{0}^{t-T_{1}(t)}\left(\frac{u_{m}^{T}(\theta) u_{m}(\theta)}{1-\dot{T}_{1}(\tau)}\right) d \theta+\frac{1}{2} \int_{0}^{t}\left(v_{s}^{T}(\tau) v_{s}(\tau)\right) d \tau \\
& -\frac{1}{2} \int_{0}^{t-T_{2}(t)}\left(\frac{v_{s}^{T}(\eta) v_{s}(\eta)}{1-\dot{T}_{2}(\tau)}\right) d \eta
\end{aligned}
$$

where $\theta=\tau-T_{1}(\tau)$ and $\eta=\tau-T_{2}(\tau)$. Note that, in the above simplifications, we are using the facts that $d \theta=d \tau-\dot{T}_{1}(\tau) d \tau$ and $d \eta=d \tau-\dot{T}_{2}(\tau) d \tau$. It can be easily shown that when $T_{1}$ and $T_{2}$ are constant, (3.2) will be positive. In this case, the communication channel's passivity is ensured in the exact same way as in the original wave formalism proposed in the literature [20].

$$
\begin{aligned}
& \text { If } \dot{T}_{1}(\tau)>1 \text { and } \dot{T}_{2}(\tau)>1 \text {, we will have } \\
& \qquad-\frac{1}{2} \int_{0}^{t-T_{1}(t)}\left(\frac{u_{m}^{T}(\theta) u_{m}(\theta)}{1-\dot{T}_{1}(\tau)}\right) d \theta>0, \quad-\frac{1}{2} \int_{0}^{t-T_{2}(t)}\left(\frac{v_{s}^{T}(\eta) v_{s}(\eta)}{1-\dot{T}_{2}(\tau)}\right) d \eta>0,
\end{aligned}
$$

which result in $\int_{0}^{t} P(\tau) d \tau>0$. On the other hand, for $\dot{T}_{1}(\tau) \leq 1$ and $\dot{T}_{2}(\tau) \leq 1$, the method proposed in [15] can be used to have a passive communication channel.

In time-delayed teleoperation systems, however, it is possible to have $\dot{T}_{1}(\tau) \geq 1$ and $\dot{T}_{2}(\tau) \leq 1$ and/or vice versa at time instants or in time periods during the teleoperation. In such cases, the communication channel's passivity is not guaranteed. In this paper, it is tried to introduce a new method and lift all assumptions on $\dot{T}_{1}(\tau)$ and $\dot{T}_{2}(\tau)$ by modeling a time-varying delay in the communication channel as a constant delay along with an external disturbance. Note that our approach will maintain the input-output relationships for each wave block remains as in (2.2). 


\section{Main Contribution}

In this part, a method is introduced to improve transparency of the proposed scheme. The basis of this part is modeling a time-varying delay in the communication channel as a constant delay along with an external disturbance. Therefore, if the disturbance can be estimated, it is possible to compensate for the tracking errors caused by the disturbance (i.e., the delay variations). In Figure 3, conventional wave variable blocks are used with constant time delays $T_{1 c}$ and $T_{2 c}$ in the feedforward and feedback paths as well as two disturbances at the outputs of the communication channel.

In order to figure out that $D_{1}(t)$ and $D_{2}(t)$ would decrease the teleoperation transparency, let us consider the tracking errors

$$
\begin{array}{ll}
\left.e_{\dot{x}}(t) \triangleq \dot{x}_{s}(t)\right|_{\dot{x}_{m}(t)=0^{\prime}} & f_{S}(t)=0, \\
\left.e_{f}(t) \triangleq f_{m}(t)\right|_{f_{s}(t)=0^{\prime}} & \dot{x}_{m}(t)=0,
\end{array}
$$

which comply with the following dynamics:

$$
\begin{aligned}
& e_{\dot{x}}(t)+e_{\dot{x}}\left(t-T_{c 1}-T_{c 2}\right)=\frac{\sqrt{2 b}}{b}\left(D_{1}(t)-D_{2}\left(t-T_{c 1}\right)\right), \\
& e_{f}(t)+e_{f}\left(t-T_{c 1}-T_{c 2}\right)=-\sqrt{2 b}\left(D_{2}(t)+D_{1}\left(t-T_{c 2}\right)\right) .
\end{aligned}
$$

By using the estimates of $D_{1}(t)$ and $D_{2}(t)$, which are denoted by $\widehat{D}_{1}(t)$ and $\widehat{D}_{2}(t)$, respectively, it is possible to decrease the tracking errors in the communication network.

Observing the proposed scheme in Figure 3, we have

$$
\begin{aligned}
u_{s}(t) & =u_{m}\left(t-T_{1 c}\right)+\left\{u_{m}\left(t-T_{1}(t)\right)-u_{m}\left(t-T_{1 c}\right)\right\}, \\
v_{m}(t) & =v_{s}\left(t-T_{2 c}\right)+\left\{v_{s}\left(t-T_{2}(t)\right)-v_{s}\left(t-T_{2 c}\right)\right\} .
\end{aligned}
$$

Therefore, it is easy to define $D_{1}(t)$ and $D_{2}(t)$ as

$$
\begin{aligned}
& D_{1}(t) \triangleq u_{m}\left(t-T_{1}(t)\right)-u_{m}\left(t-T_{1 c}\right), \\
& D_{2}(t) \triangleq v_{s}\left(t-T_{2}(t)\right)-v_{s}\left(t-T_{2 c}\right) .
\end{aligned}
$$

In order to estimate $D_{1}(t)$ and $D_{2}(t)$, first of all, we have to estimate $u_{m}\left(t-T_{1 c}\right)$ and $v_{s}\left(t-T_{2 c}\right)$. Note that $u_{m}\left(t-T_{1}(t)\right)$ and $v_{s}\left(t-T_{2}(t)\right)$ can be measured directly in the proposed scheme. Therefore

$$
\begin{aligned}
& \widehat{D}_{1}(t)=u_{m}\left(t-T_{1}(t)\right)-\widehat{u}_{m}\left(t-T_{1 c}\right), \\
& \widehat{D}_{2}(t)=v_{s}\left(t-T_{2}(t)\right)-\widehat{v}_{s}\left(t-T_{2 c}\right) .
\end{aligned}
$$

Let us inspect the estimation of $\widehat{u}_{m}\left(t-T_{1 c}\right)$ which is applicable for the estimation of $\widehat{v}_{s}\left(t-T_{2 c}\right)$. Since we can just measure $u_{m}\left(t-T_{1}(t)\right)$ and we need the estimation of $u_{m}\left(t-T_{1 c}\right)$, 


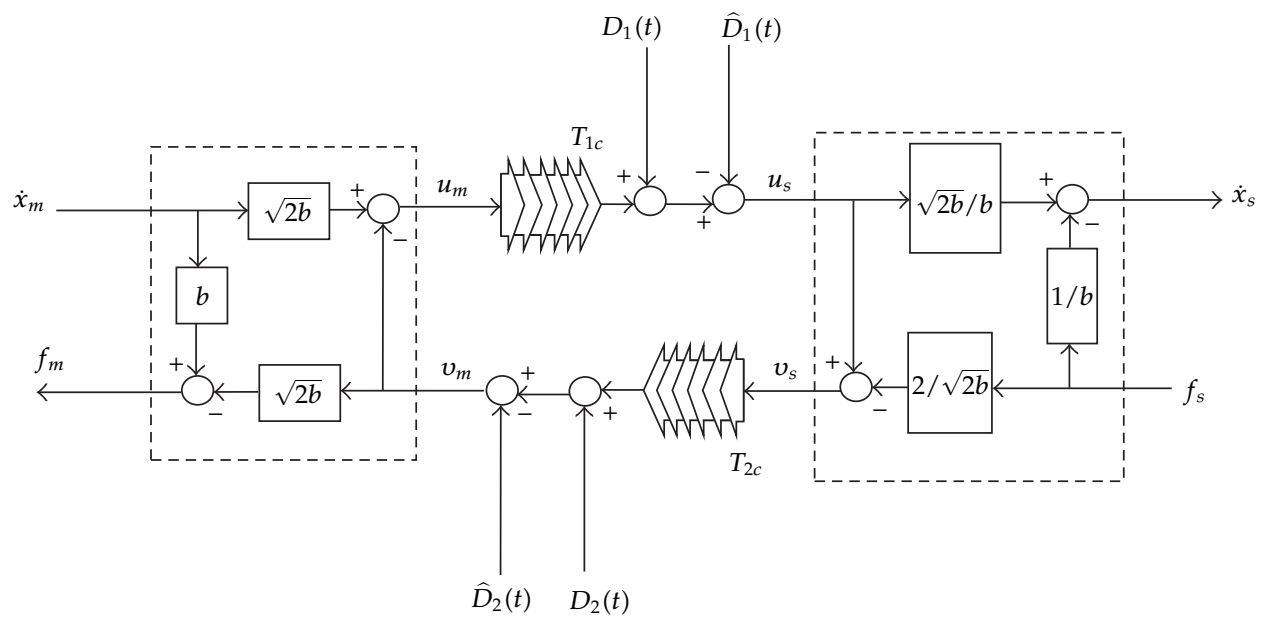

Figure 3: Conventional wave variable scheme with disturbances and their estimations in the communication channel.

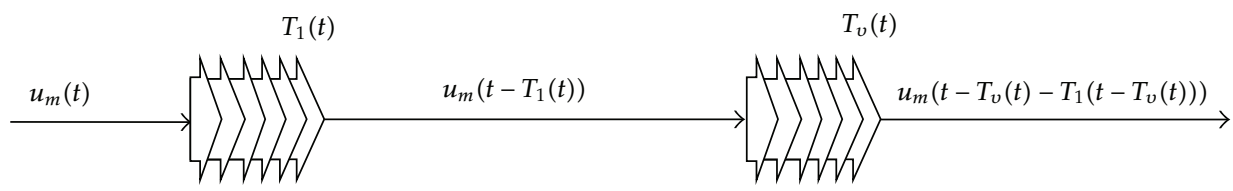

Figure 4: Real communication delay combined with virtual delay.

in which $T_{1 c}$ is constant, the proposed method is to add a virtual delay, $T_{v}(t)$, in the estimating block as in Figure 4.

The virtual delay, $T_{v}(t)$, should be defined in such a way that $u_{m}\left(t-T_{v}(t)-T_{1}\left(t-T_{v}(t)\right)\right)$ would be equal to $u_{m}\left(t-T_{1 c}\right)$ :

$$
T_{v}(t)+T_{1}\left(t-T_{v}(t)\right)=T_{1 c}
$$

Therefore, to calculate the required amount of the virtual delay $T_{v}(t)$ such that (4.6) holds, we need to be able to measure $T_{1}(t)$. Based on the scheme in Figure 4 which has been used in [21], $T_{1}(t)$ can be found.

To have a causal virtual delay $T_{v}(t)$, that is, $T_{v}(t)>0$, it could be assumed that $T_{1 c}=$ $T_{\max }+T_{0}$ where $T_{\max } \triangleq \sup _{t} T_{1}(t)$ and $T_{0} \triangleq T_{1}(0)$. A geometric approach to estimate $T_{v}(t)$ is studied in the appendix. Using the estimated virtual delay, it is possible to estimate $\widehat{u}_{m}\left(t-T_{1 c}\right)$ and then $\widehat{D}_{1}(t)$.

The overall proposed scheme is shown in Figure 6, in which a disturbance estimator block in each of the feedforward and feedback paths is added to compensate for the variable time delay effects in terms of velocity and force tracking performance.

Adding $\widehat{D}_{1}(t)$ and $\widehat{D}_{2}(t)$ in the proposed scheme, time-varying delays in the communication channel effectively act as constant delays. Therefore, the overall proposed method shown in Figure 6 would have better stability and transparency behaviors. 


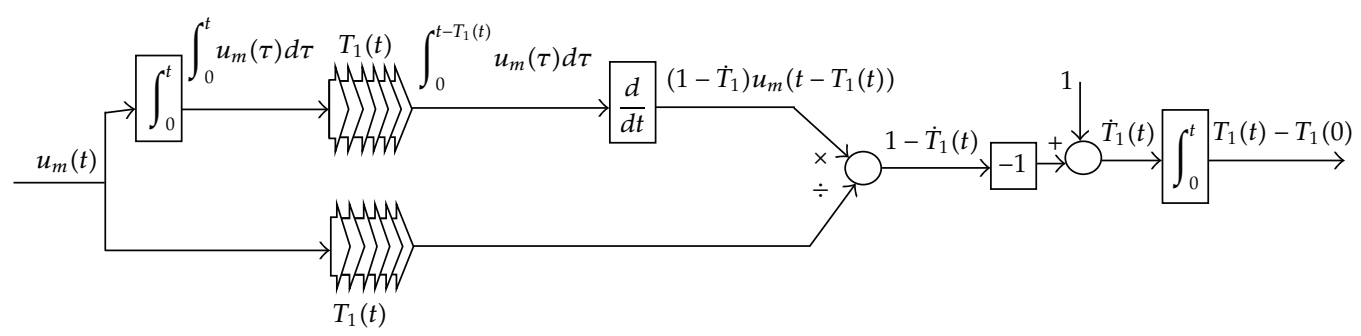

Figure 5: Estimating block for $T_{1}(t)$ that added by an unknown constant.

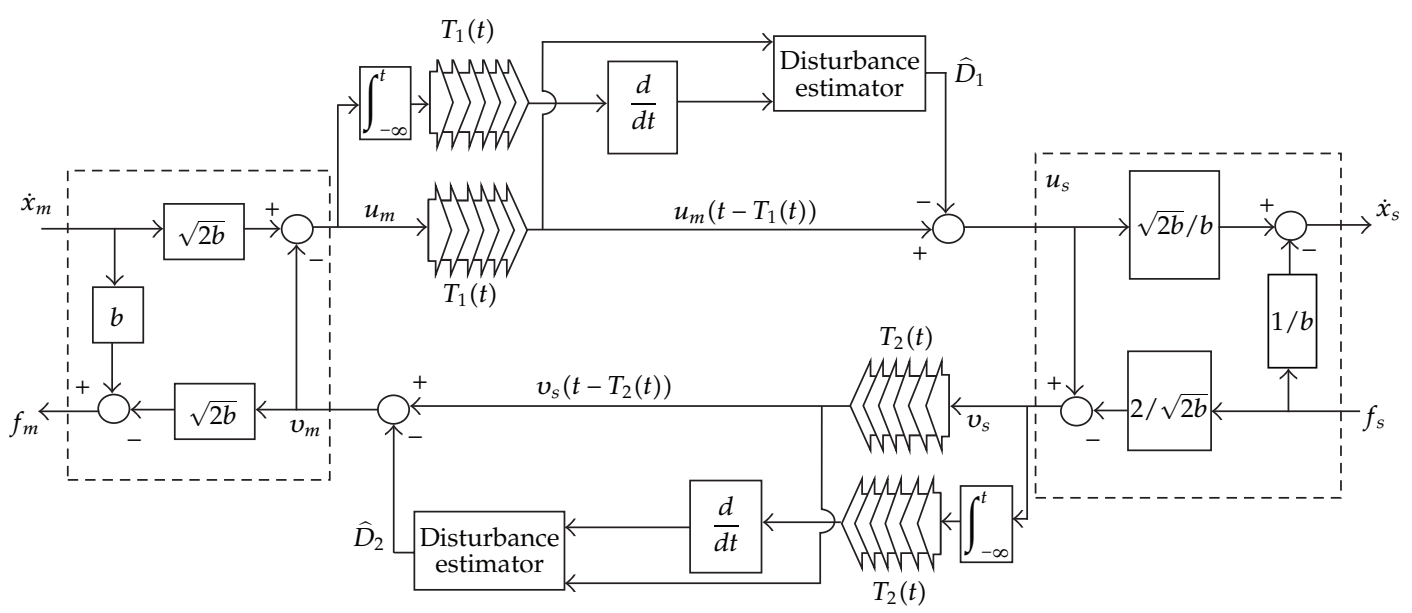

Figure 6: The overall proposed scheme.

\section{Simulation Results}

In this part, simulation results of the proposed method in controlling of the PHANToM robot in telerobotics application are studied. In the later parts, we first study the PHANToM robot and then apply simple $\mathrm{P}+\mathrm{D}$. controller to the robots in telemanipulation with the proposed scheme.

\subsection{PHANTOM Setup}

The schematics of PHANToM robot with its corresponding joint angles are shown in Figure 7. The local and remote PHANToM dynamics can be modeled as

$$
\begin{gathered}
M_{l}\left(q_{l}\right) \ddot{q}_{l}+C_{l}\left(q_{l}, \dot{q}_{l}\right) \dot{q}_{l}+N_{l}\left(q_{l}\right)=\tau_{h}-\tau_{l}, \\
M_{r}\left(q_{r}\right) \ddot{q}_{r}+C_{r}\left(q_{r}, \dot{q}_{r}\right) \dot{q}_{r}+N_{r}\left(q_{r}\right)+J_{r}^{T}\left(q_{r}\right) F_{e}=\tau_{r},
\end{gathered}
$$

where $q_{i}, \dot{q}_{i}$ and $\ddot{q}_{i}$ for $i \in\{r, l\}$ are the joint positions, velocities, and accelerations of the local and the remote robots, respectively, and $J_{r}^{T}\left(q_{r}\right) F_{e}$ is the contact effect between remote robot and environment. The local and remote PHANToM dynamics are given in [22]. 


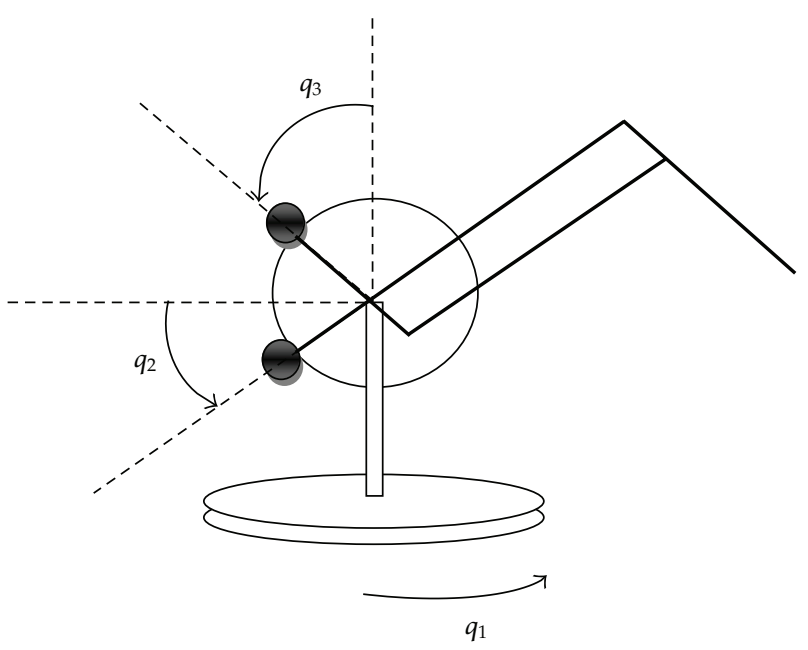

Figure 7: Schematics of PHANToM robot with its corresponding joint angles.

\subsection{Simulation with Hard Contact Scenario}

The overall block diagram is shown in Figure 8. In this controlling system, proposed scheme shown in Figure 6 is used to decode and send velocity and force signals to the other side. To have more suitable performance of position tracking, integrals of wave variables are employed [23]. This way it is possible to send integrals of velocity and force, that is, position and momentum, which makes it possible to avoid the effects of mismatch in initial position to the performance of position tracking. Following controllers used in local and remote sides are simple $\mathrm{P}+\mathrm{D}$ controllers added by gravitational term and measured environment force:

$$
\begin{gathered}
\tau_{l}=K_{P} q_{l}+K_{D} \dot{q}_{l}-N_{l}\left(q_{l}\right)+J_{l}^{T}\left(q_{l}\right) f_{m}, \\
\tau_{r}=-K_{P}\left(q_{r}-x_{s}\right)-K_{D} \dot{q}_{r}+N_{r}\left(q_{r}\right),
\end{gathered}
$$

where $f_{m}$ is the force command transmitted to the local side and $x_{s}$ is the position command transmitted to the remote side to be used in the local and remote controllers, respectively (see Figure 8). Also, $J_{l}^{T}\left(q_{l}\right)$ is the Jacobin transpose of the local robot and used to transform a Cartesian-space force to its equivalent joint-space torque. In the controller (5.2), the parameters $K_{P}$ and $K_{D}$ are proportional and derivative matrix gains that can be adjusted by the user. In this simulation, the proportional gain $K_{P}$ is set to $3 I$, and the derivative gain $K_{D}$ is set to $1.5 I$, where $I$ is the identity matrix.

Human force $F$ is applied to the local robot in $X$-direction as shown in Figure 9. Human force is 0 between 0 and $1 \mathrm{~s}$ and increases to $4 \mathrm{~N}$ at $2.5 \mathrm{~s}$. The force is kept constant until $9.5 \mathrm{~s}$ then increases to $10 \mathrm{~N}$ at $12 \mathrm{~s}$. It is kept constant until $27.5 \mathrm{~s}$ and then decreases to $4 \mathrm{~N}$ at $30.5 \mathrm{~s}$ and remains constant between $30.5 \mathrm{~s}$ and $40 \mathrm{~s}$.

In the remote side there is a stiff wall in $x=0.3 \mathrm{~m}$. When the remote robot reaches the wall and moves further, the feedback force $1000(x-0.3) \mathrm{N}$ is applied to the remote robot. In this simulation, the initial conditions are set to $q_{l}(0)=\dot{q}_{l}(0)=q_{r}(0)=\dot{q}_{r}(0)=\left[\begin{array}{lll}0 & 0 & 0\end{array}\right]^{T}$, and it is assumed that time delays are sinusoidal. The simulation results are shown in Figures 10, $11,12,13$, and 14. Figures 10, 11, and 12 show the $X_{-}^{-}, Y_{-}$-, and Z-positions of the local and the 


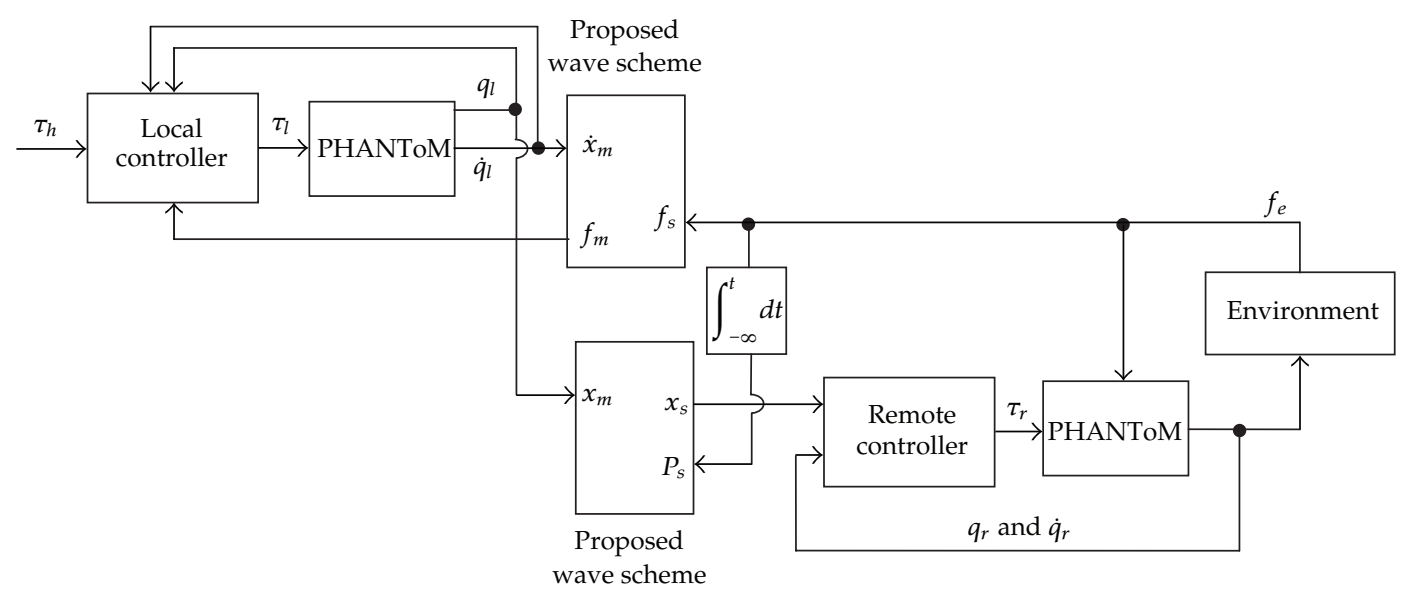

Figure 8: The overall controlling block diagram.

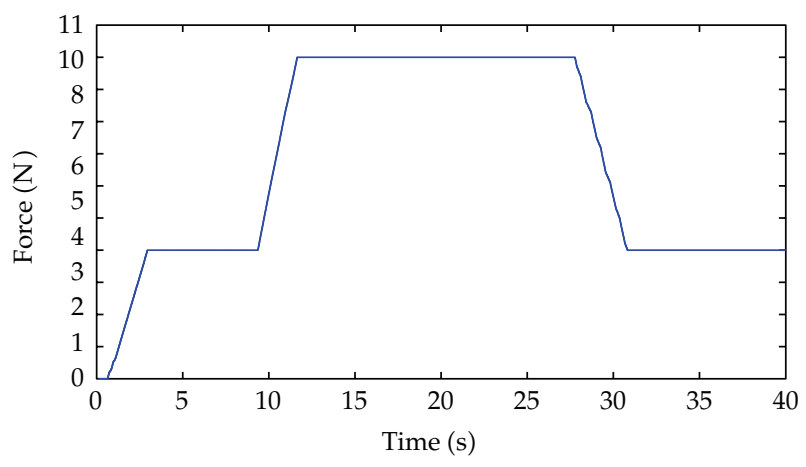

Figure 9: Human force applied at local robot in $x$-direction.

remote robots. Figure 13 shows the environment force, $F_{e}$, and the force applied to the local robot, $F_{m}$, from the communication channel. The force $F_{m}$ is the force that could be sensed by human when remote robot contacts an obstacle.

When human forces increases, the local and remote robots move forward that can be seen from Figure 10. About 14 seconds, the remote robot contacts the wall at $x=0.3 \mathrm{~m}$ and could not move longer, while the local robot moves further because of the human force and reaches about $0.4 \mathrm{~m}$. In Figure 13, it could be seen that an environment force applied to the remote robot at about instant 14 when remote robot touches the wall. Environment force increases rapidly and after some damping oscillation reaches a constant value $1 \mathrm{~N}$. It is noticeable that $F_{m}$ has similar behavior as $F_{e}$, and it means that human senses same force as environment force which is applied to the remote robot by the wall. About time $30 \mathrm{~s}, F_{e}$ and $F_{m}$ reduce to zero which means that remote robot is taken away from the wall and both robot's positions act synchronously between $35 \mathrm{~s}$ and $40 \mathrm{~s}$ which could be seen from Figure 10.

In this simulation, human force is applied just in $X$-direction but because of coupling effects in PHANToM dynamics, some little movements happen in $Y$ - and Z-directions which are shown in Figures 11 and 12. 


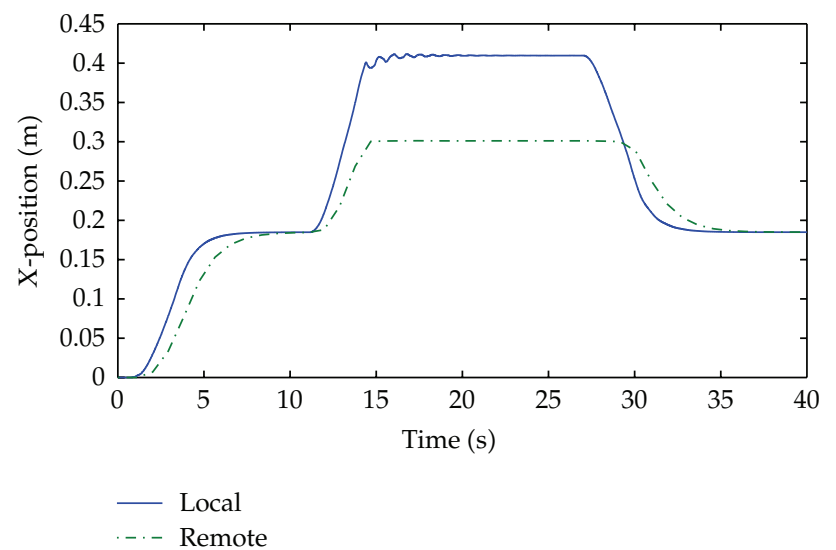

Figure 10: $X$-position of the local and remote robots.

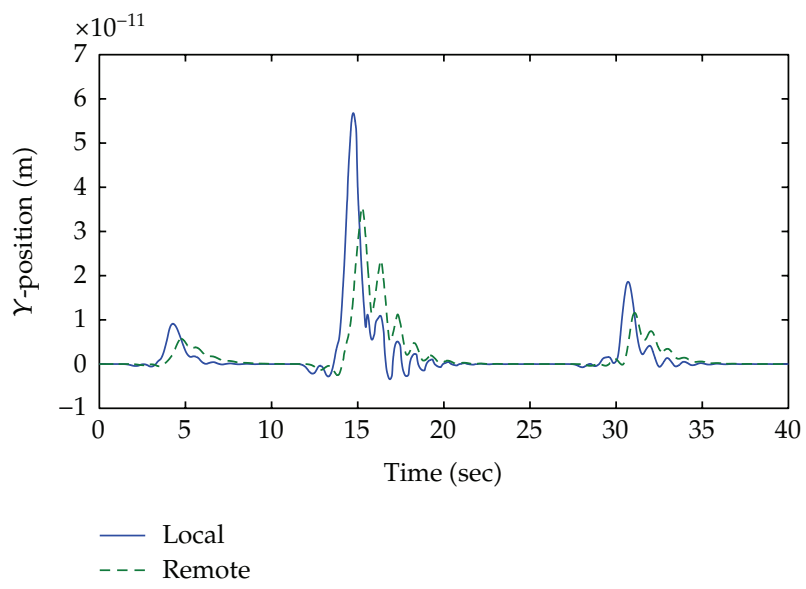

Figure 11: $Y$-position of the local and remote robots.

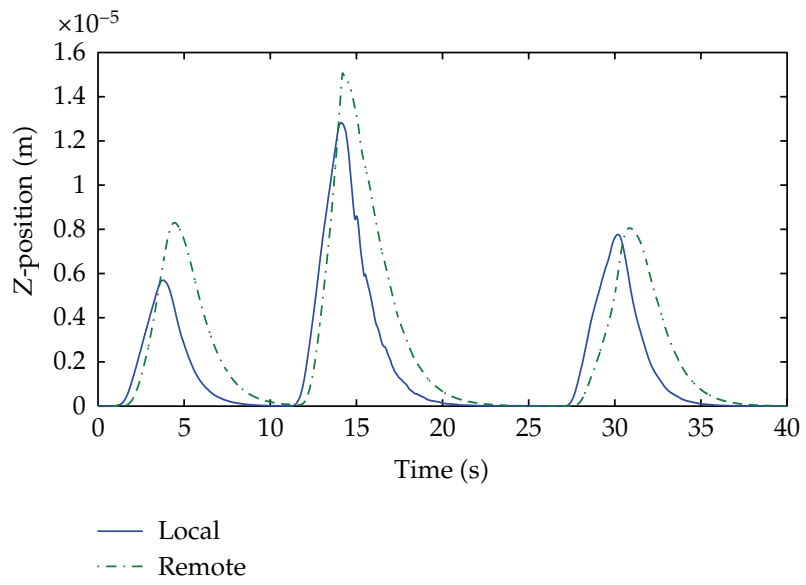

Figure 12: Z-position of the local and remote robots. 


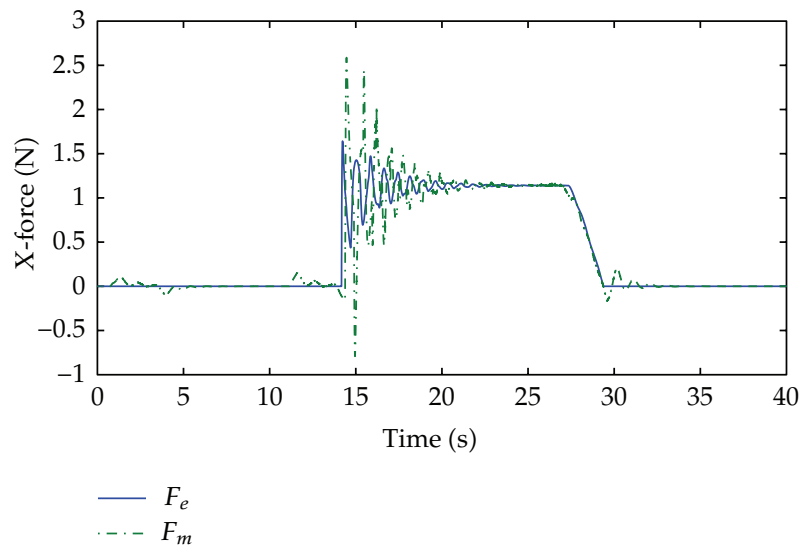

Figure 13: Environment force, $F_{e}$, and the force sensed by human, $F_{m}$, in $X$-direction.

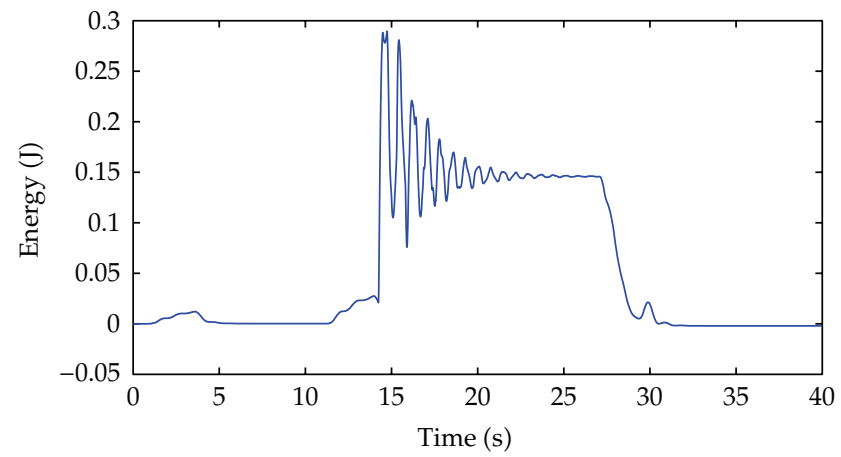

Figure 14: The absorbed energy in the communication channel.

To study passivity simulation of the proposed method, the net energy exchange at the two ports of the communication channel, $\int_{0}^{t} \dot{x}_{m}(\tau) F_{m}(\tau)-\dot{x}_{s}(\tau) F_{s}(\tau) d \tau$, is shown in Figure 14 .

Considering Figure 14, it could be seen that before and after contacts between remote robot and the environment, the net exchange energy is almost zero. During contact between $14 \mathrm{~s}$ and $30 \mathrm{~s}$, it could be seen from Figure 14 that about 0.12 joules energy is absorbed in the communication channel. Regarding the fact that $F_{m}$ is almost equal to $F_{s}$, most of absorbed energy is because of the differences between $x_{m}$ and $x_{s}$ during contact.

\section{Experimental Results}

To verify the theoretical results presented in the paper, the local and the remote manipulators are considered to be two PHANToM Omni robots (Sensable Technologies, Inc., Wilmington, MA) as shown in Figure 17. The utilized PHANToM devices are three degree-of-freedom robots that map the generalized joint angles of the robot $\left(q_{1}, q_{2}\right.$, and $\left.q_{3}\right)$ to the Cartesian position $(X, Y$, and $Z)$ of the gimbal. The local and remote robots are connected to a computer using FireWire IEEE 1394 ports. One robot (master) interacts with the human hand while the other one (slave) interacts with an environment. This experiment will compare the stability and performance of the proposed method versus the conventional wave variable scheme. 


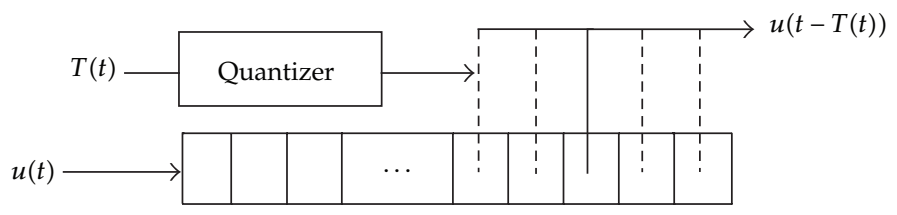

Figure 15: Implementation of the time-varying time delay for the experiments.

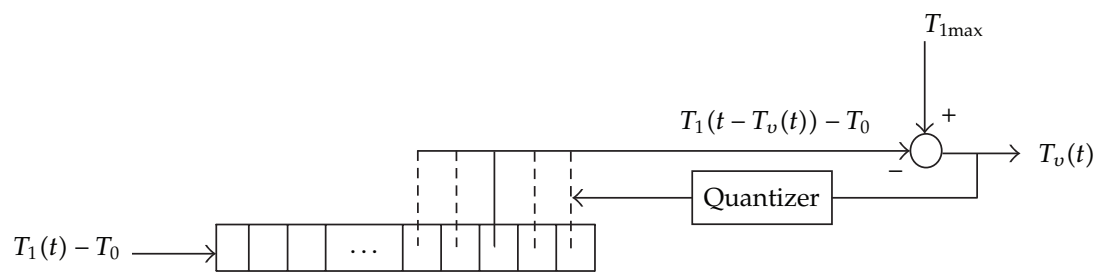

Figure 16: The implementation of the scheme to estimate $T_{v}(t)$.

Controllers for the local and the remote robots are implemented based on (5.2). In this experiment, the proportional gain $K_{P}$ and the derivative gain $K_{D}$ are set to $3 I$ and $1.5 I$, respectively, in which $I$ is the identity matrix. To artificially create time-varying time delays between the local and the remote robots, a first-input/first-output delay-inducing buffer is used for each robot-changing the position of the indicator, which is related to the present-time delay's value, causes this buffer to simulate time-varying time delays in the communication channel (Figure 15).

To implement the virtual delay $T_{v}(t)$, a scheme similar to the block diagram shown in Figure 23 in the appendix is used; see Figure 16. In this scheme, the input $T_{1}(t)-T_{0}$ is obtained from the system shown in Figure 5. Note that the Quantizer block in Figures 15 and 16 is used to discretize the time delay; the quantized time delay value will determine the number of buffer cells required at each sampling time.

The schematics of the PHANToM robot with its joint angles are shown in Figure 7. The dynamics of the local and remote PHANToM robots are same as (5.1), and the block diagram of the experimental teleoperation system is shown in Figure 8. The proposed scheme is used to transform and send velocity and force signals from one end to the other end of the teleoperation system. Integrals of wave variables are also transmitted to avoid an offset in position tracking. The controllers (5.2) used for the local and remote robots have a P+D term and a gravity compensation term. The local robot controller also has a force term. The choice of controllers is not unique; the selection (5.2) for controllers is only used to test the passivity of the communication channel and compare the proposed method with the conventional wave variable scheme in time-varying delay teleoperation.

In this experiment, the forward and backward time delays are chosen to be the asymmetric sinusoidal time-varying functions $T_{1}(t)=0.11(1+\sin 10 t)$ and $T_{2}(t)=0.11(1+$ $\cos 10 t)$. Note that the time derivatives of these varying time delays are in the range $0 \leq \dot{T} \leq$ 1.1. The fact that $\dot{T}$ exceeds 1 makes it possible to show the effectiveness of the proposed method for fast varying time delays as compared to past methods.

To study the passivity of the proposed method compared with the typical wave variable method, the net energy exchange at the two ports of the communication channel, $\int_{0}^{t} \dot{x}_{m}(\tau) F_{m}(\tau)-\dot{x}_{s}(\tau) F_{s}(\tau) d \tau$, is shown in Figures 18 and 19. Whereas in Figure 18 (i.e., in the 


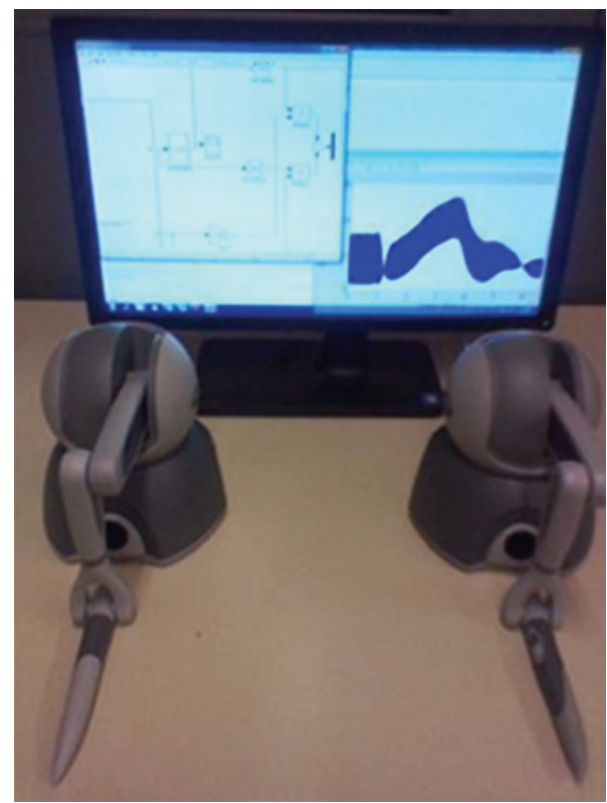

Figure 17: Two PHANToM robots connected to the teleoperation system.

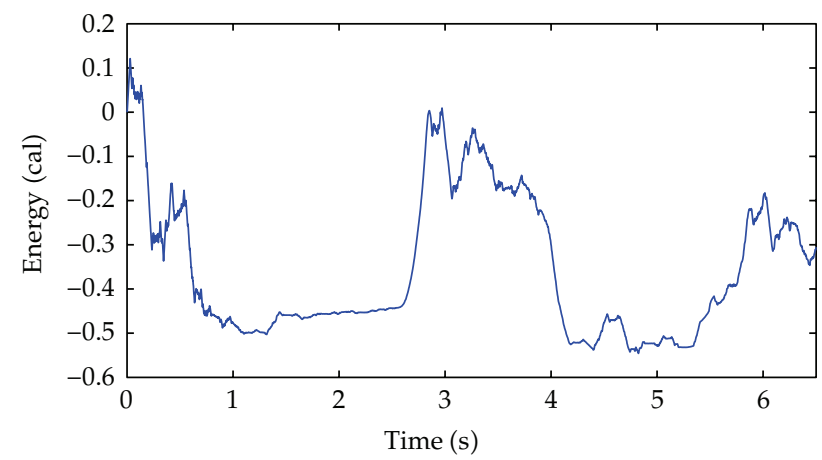

Figure 18: The energy in the communication channel in the conventional wave variable scheme.

conventional wave variable scheme) the energy in the channel becomes negative at certain times, which means that the communication channel produces energy and is nonpassive, in Figure 19 (i.e., in the proposed scheme) the energy in the channel is always positive.

To compare the tracking performance under the proposed method and the conventional wave variable scheme in the presence of varying time delays, experimental results concerning joint positions tracking between the local and the remote robots using the same controllers are shown in Figures 20 and 21. It is evident from Figure 21 that instability happens in the teleoperation system with the conventional wave variables in the presence of time-varying delay. Using the proposed method, however, position tracking is considerably better in the presence of the same variable time delays-see Figure 20.

Note that the single-way time delays used in this experiment vary between 0 and $220 \mathrm{~ms}$ with a frequency of $10 \mathrm{~Hz}$. This is reasonable in Internet-based teleoperation 


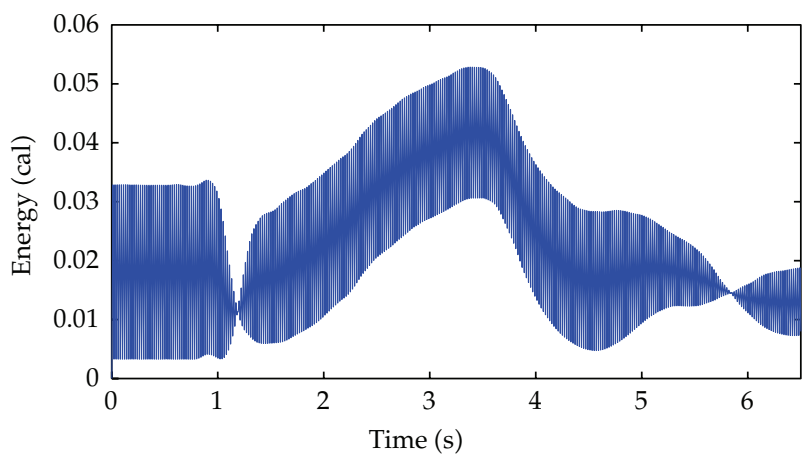

Figure 19: The energy in the communication channel using the proposed method.

applications because the round-trip delay has been shown to have an average of $350 \mathrm{~ms}$ if the distance between the local and the remote robots is up 10,000 km [24]. Increasing the time delay further might cause the teleoperation system to be unstable; it is well known that wave reflection [6] can cause stability problems under large delays.

Also note that with a different choice of the control laws, these results may change; yet our contribution in terms of passivating the communication channel under varying delays is independent of that.

\section{Conclusion and Future Works}

In this paper a new method is proposed to pacify communication channels with varying time delays. Using proposed method, the effect of varying delays on the transmitted signals could be seen as an additive disturbance to the delayed signals which is transmitted in the channel with constant time delay. By defining a virtual delay, this disturbance is estimated and added to the channel with negative sign so that the effect of varying time delay on the transmitted signal reduces. In fact, using disturbance estimator and combining it by time-varying delay in the communication channel it is possible to make the channel seen as a constant time delay. The efficiency of the proposed method is studied in simulation and experiment in which two PHANToM robots are connected via communication network with time varying delays.

In the scheme shown in Figure 5, the differentiation and integration blocks may cause noisy signals and noise accumulation, respectively. By frequently resetting the integrator, the noise accumulation problem can be alleviated. Also, it is possible to use differentiators that are robust to measurement noise. For instance, Levant [25] designed a robust exact differentiator, and Suzuki et al. [26] proposed an adaptive version of Levant's differentiator. Incorporating such robust differentiators in our scheme remains as future work.

\section{Appendices}

Two methods for estimation of the virtual delay $T_{v}(t)$ are proposed here. We name the first method as geometric and the second one as block diagram based. 


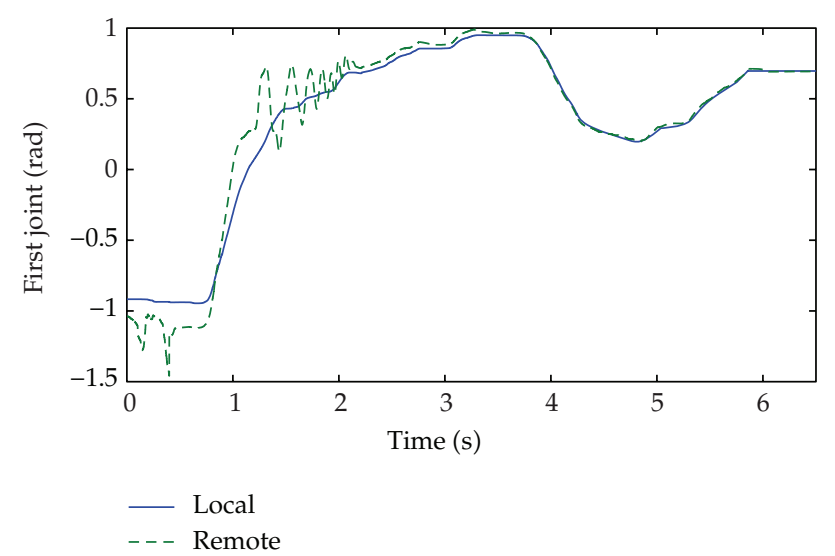

(a)

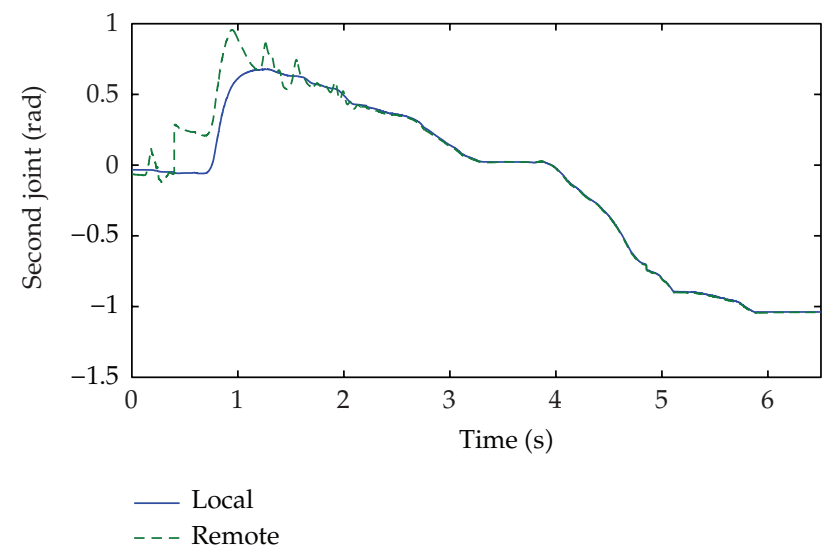

(b)

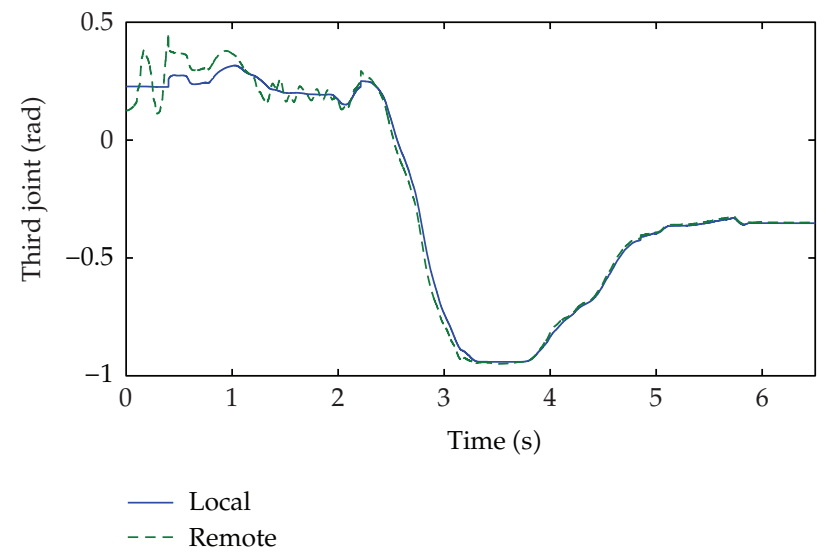

(c)

Figure 20: Joint tracking between the local and remote robots during teleoperation using the proposed scheme. 


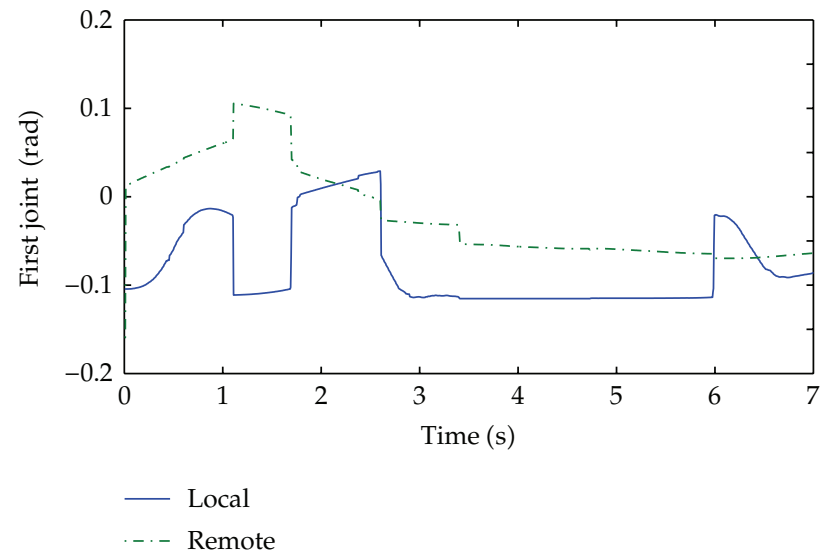

(a)

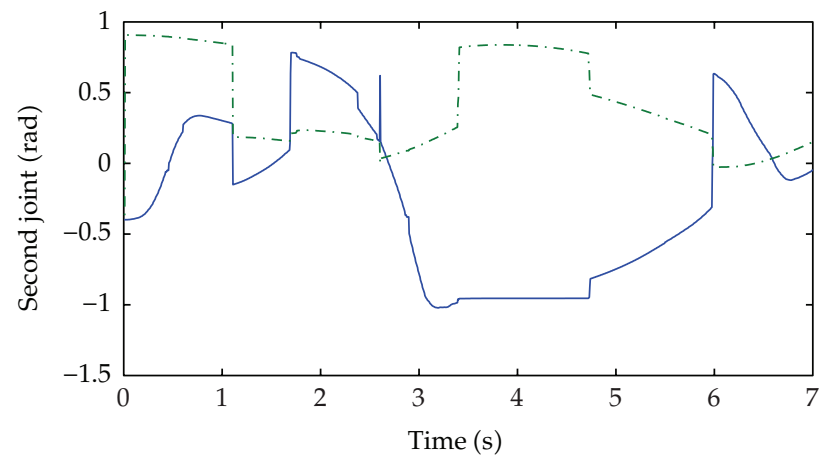

- Local

-. - Remote

(b)

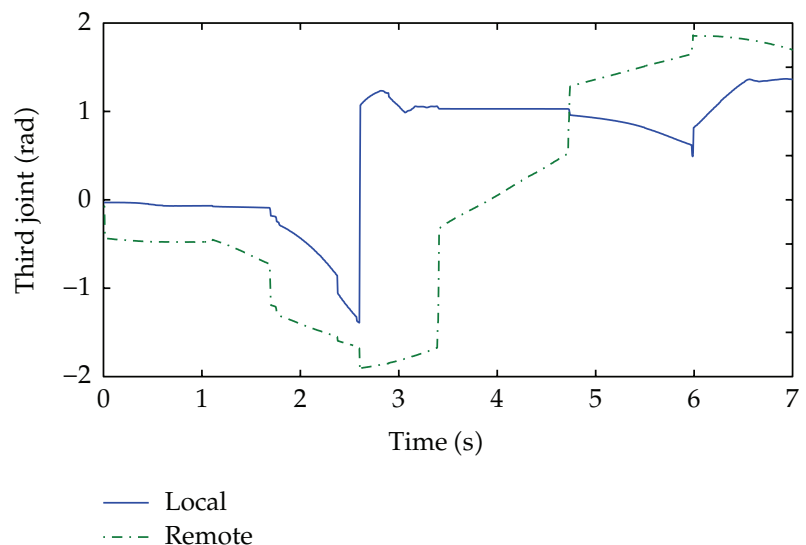

(c)

Figure 21: Joint tracking between the local and remote robots during teleoperation using the conventional wave variable scheme. 


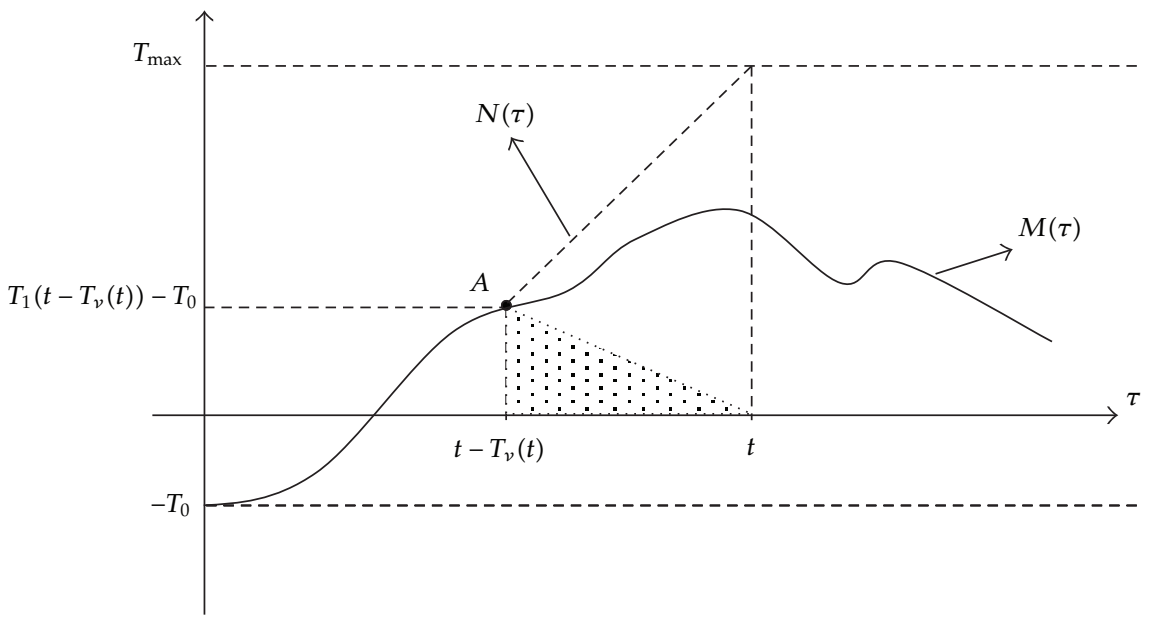

Figure 22: A typical profile of $M(\tau)$ and $N(\tau)$.

\section{A. Geometric Approach to Estimate the Virtual Delay $T_{v}(t)$}

Inspired by the method used in [27], we will investigate how (4.6) can be used to estimate $T_{v}(t)$ geometrically. From Figure 5 , we can estimate $T_{1}(t)-T_{0}$; thus define $M(\tau) \triangleq$ $T_{1}(\tau)-T_{0}$ and assume it has a typical profile of time-varying time delays as shown in Figure 22. Here, $\tau$ is the general time variable and $t$ is the current instant.

Using the definition of $M(\tau)$, at time $\tau=t-T_{v}(t)$, we have $M(\tau)=T_{1}\left(t-T_{v}(t)\right)-$ $T_{0}$, which corresponds to point $A$ in Figure 22. Note that in the shaded triangle shown in Figure 22, the horizontal side's length is $T_{v}(t)$ and the vertical side's length is $T_{1}\left(t-T_{v}(t)\right)-T_{0}$. Therefore, to estimate $T_{v}(t)$, a point $A$ in the profile $M(\tau)$ should be found in such a way that the sum of the vertical and horizontal sides of the triangle equals $T_{\max }$; that is, we need to have $\left\{T_{v}(t)\right\}+\left\{T_{1}\left(t-T_{v}(t)\right)-T_{0}\right\}=T_{\max }$.

We know from trigonometry that if the sum of vertical and horizontal sides in a rightangle triangle is constant, then the locus of each vertex is a straight line. Therefore, as the horizontal side's length decreases, the vertical side's length increases and the vertex moves on the straight line $N(\tau)$. At time $\tau=t$, when the horizontal side vanishes, the vertical side's length will be $T_{\max }$.

We have $N(\tau) \triangleq \tau+\left(T_{\max }-t\right)$ because it interconnects the two points $\left(t-T_{v}(t), T_{1}(t-\right.$ $\left.\left.T_{v}(t)\right)-T_{0}\right)$ and $\left(t, T_{\max }\right)$. Since the point $A$ is the intersection of the two profiles $M(\tau)$ and $N(\tau)$, we have

$$
M(\tau)=N(\tau) \stackrel{\text { yields }}{\longrightarrow} T_{1}(\tau)-T_{0}=\tau+\left(T_{\max }-t\right) \stackrel{\text { yields }}{\longrightarrow} T_{1}(\tau)-T_{0}=T_{\max }-(t-\tau) .
$$

Note that using the scheme shown in Figure 5, it is possible to estimate $T_{1}(\tau)-T_{0}$ for every instant $\tau \leq t$. Using the estimated profile $\widehat{T}_{1}(\tau)-T_{0}$ for past times $\tau \leq t$, at a given time $t$, the nonlinear equation $\widehat{T}_{1}\left(\tau_{A}\right)-T_{0}=T_{\max }-\left(t-\tau_{A}\right)$ can be solved numerically and $\tau_{A}$ can be found. Then the amount of the virtual delay $\widehat{T}_{v}(t)=t-\tau_{A}$ is found, which is what we strived to determine. 


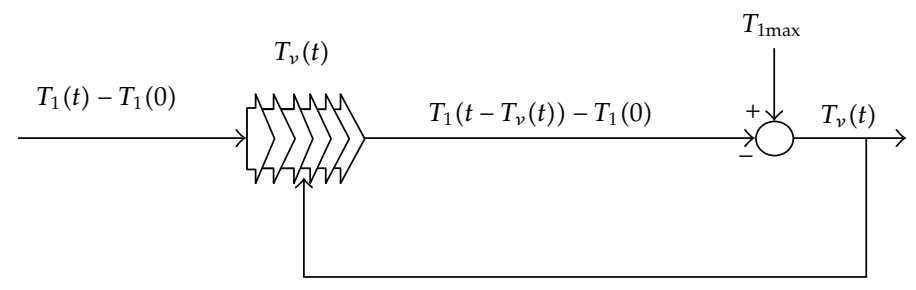

Figure 23: Alternative scheme to estimate $T_{v}(t)$.

\section{B. Block Diagram Approach to Estimate the Virtual Delay $T_{v}(t)$}

To estimate the virtual delay $T_{v}(t)$, we can alternatively use the scheme shown in Figure 23 in which $T_{1}(t)-T_{0}$ is the output of the scheme shown in Figure 5. Note that the scheme shown in Figure 23 is readily implementable in environments such as Simulink where time-varying time delay blocks exist.

\section{References}

[1] T. B. Sheridan, "Space teleoperation through time delay: review and prognosis," IEEE Transactions on Robotics and Automation, vol. 9, no. 5, pp. 592-606, 1993.

[2] B. Hannaford, "A design framework for teleoperators with kinesthetic feedback," IEEE Transactions on Robotics and Automation, vol. 5, no. 4, pp. 426-434, 1989.

[3] T. Imaida, Y. Yokokohji, T. Doi, M. Oda, and T. Yoshikawa, "Ground-space bilateral teleoperation of ETS-VII robot arm by direct bilateral coupling under 7-s time delay condition," IEEE Transactions on Robotics and Automation, vol. 20, no. 3, pp. 499-511, 2004.

[4] R. J. Anderson and M. W. Spong, "Asymptotic stability for force reflecting teleoperators with time delay," in IEEE International Conference on Robotics and Automation, pp. 1618-1625, May 1989.

[5] G. Niemeyer and J. J. E. Slotine, "Stable adaptive teleoperation," IEEE Journal of Oceanic Engineering, vol. 16, no. 1, pp. 152-162, 1991.

[6] G. Niemeyer and J. E. Slotine, "Telemanipulation with time delays," International Journal of Robotics Research, vol. 23, no. 9, pp. 873-890, 2004.

[7] R. Lozano, N. Chopra, and M. W. Spong, "Passivation of force reflecting bilateral teleoperators with time varying delay," in Proceedings of the Proceedings of the 8th Mechatronics Forum, pp. 954-962, June 2002.

[8] S. Leeraphan, T. Maneewarn, and D. Laowattana, "Stable adaptive bilateral control of transparent teleoperation through time-varying delay," in Proceedings of the International Conference on Intelligent Robots and Systems (IEEE/RSJ '02), vol. 3, pp. 2979-2984, October 2002.

[9] D. Lee and M. W. Spong, "Passive bilateral control of teleoperators under constant time-delay," in Proceedings of the 16th Triennial World Congress of International Federation of Automatic Control (IFAC '05), vol. 16, pp. 109-114, July 2005.

[10] C. A. Desoer and M. Vidyasagar, Feedback Systems: Input-Output Properties, Academic Press, New York, NY, USA, 1975.

[11] P. F. Hokayem and M. W. Spong, "Bilateral teleoperation: an historical survey," Automatica, vol. 42, no. 12, pp. 2035-2057, 2006.

[12] S. Dashkovskiy, B. S. Rüffer, and F. R. Wirth, "An ISS small gain theorem for general networks," Mathematics of Control, Signals, and Systems, vol. 19, no. 2, pp. 93-122, 2007.

[13] S. Dashkovskiy, M. Kosmykov, and F. R. Wirth, "A small-gain condition for interconnections of ISS systems with mixed ISS characterizations," IEEE Transactions on Automatic Control, vol. 56, no. 6, pp. 1247-1258, 2011.

[14] I. G. Polushin and S. Dashkovskiy, "A small gain framework for networked cooperative teleoperation," in Proceedings of the 8th IFAC Symposium on Nonlinear Control Systems, pp. 90-95, 2010.

[15] Y. Gu, C. Zhang, and K. T. Chong, "Adaptive passive control with varying time delay," Simulation Modelling Practice and Theory, vol. 18, no. 1, pp. 1-8, 2010. 
[16] R. J. Anderson and M. W. Spong, "Asymptotic stability for force reflecting teleoperators with time delay," International Journal of Robotics Research, vol. 11, no. 2, pp. 135-149, 1992.

[17] Y. Yokokohji and T. Yoshikawa, "Bilateral control of master-slave manipulators for ideal kinesthetic coupling-formulation and experiment," IEEE Transactions on Robotics and Automation, vol. 10, no. 5, pp. 605-619, 1994.

[18] G. Niemeyer and J. J. E. Slotine, "Using wave variables for system analysis and robot control," in Proceedings of the IEEE International Conference on Robotics and Automation (ICRA '97), pp. 1619-1625, April 1997.

[19] G. Niemeyer, Using wave variables in time delayed force reflecting teleoperation, Ph.D. thesis, Department of Aeronautics and Astronautics, Massachusetts Institute of Technology, 1996.

[20] G. Niemeyer and J. J. E. Slotine, "Using wave variables for system analysis and robot control," in Proceedings of the IEEE International Conference on Robotics and Automation (ICRA '97), pp. 1619-1625, April 1997.

[21] H. Kawada and T. Namerikawa, "Bilateral control of nonlinear teleoperation with time varying communication delays," in Proceedings of the American Control Conference (ACC '08), pp. 189-194, June 2008.

[22] M. C. Cavusoglu and D. Feygin, "Kinematics and dynamics of phantom model 1.5 haptic interface," UC Berkeley ERL Memo M01/15, 2001.

[23] G. Niemeyer and J. E. Slotine, "Telemanipulation with time delays," International Journal of Robotics Research, vol. 23, no. 9, pp. 873-890, 2004.

[24] R. Oboe, "Force-reflecting teleoperation over the internet: the JBIT project," Proceedings of the IEEE, vol. 91, no. 3, pp. 449-462, 2003.

[25] A. Levant, "Robust exact differentiation via sliding mode technique," Automatica, vol. 34, no. 3, pp. 379-384, 1998.

[26] S. Suzuki, K. Furuta, and S. Shiratori, "Adaptive impact shot control by pendulum-like juggling system," JSME International Journal C, vol. 46, no. 3, pp. 973-981, 2003.

[27] L.A. Zadeh, "Operational analysis of variable-delay systems," in Proceedings of the I.R.E., pp. 254-268, May 1952. 


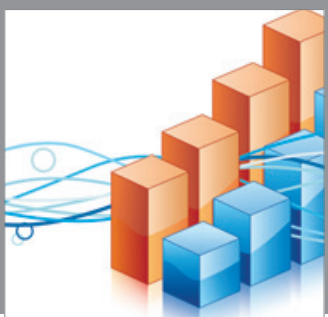

Advances in

Operations Research

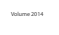

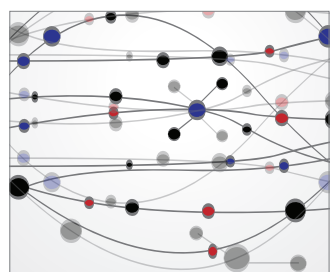

\section{The Scientific} World Journal
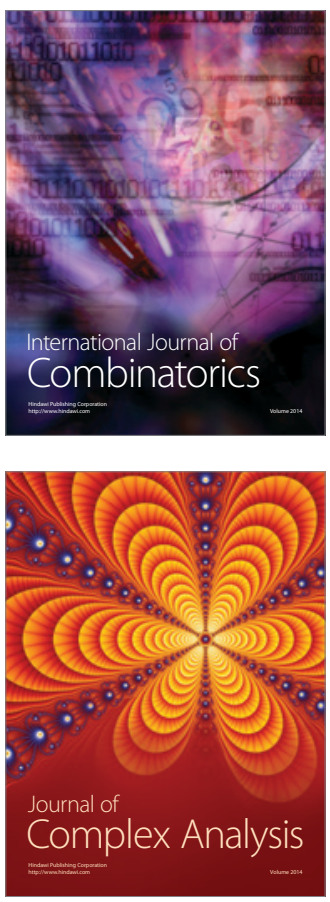

International Journal of

Mathematics and

Mathematical

Sciences
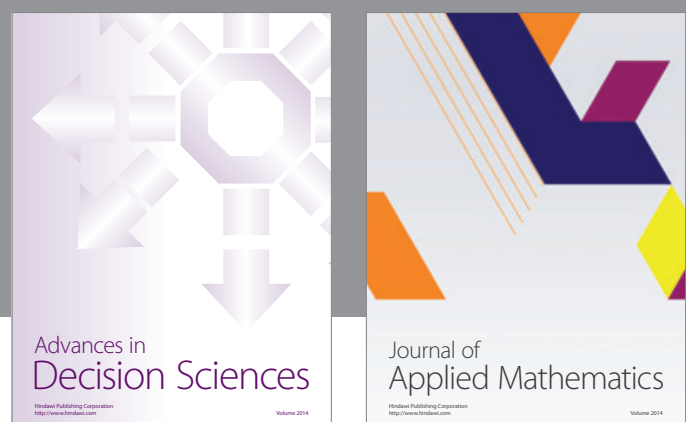

Journal of

Applied Mathematics
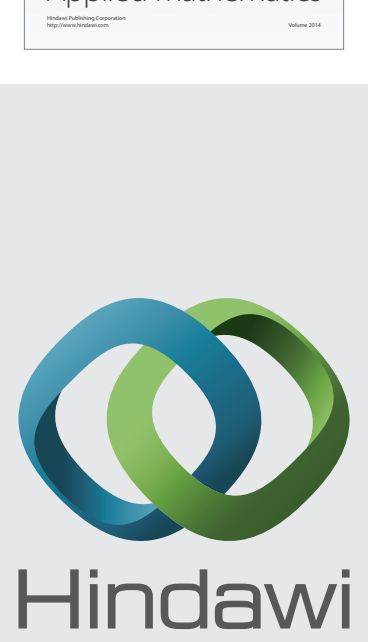

Submit your manuscripts at http://www.hindawi.com
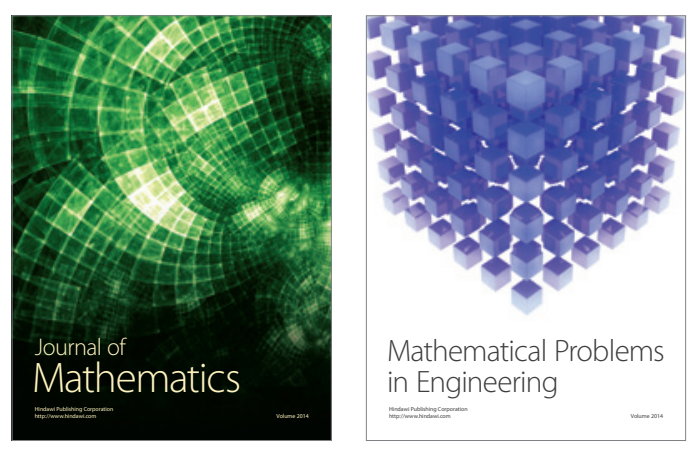

Mathematical Problems in Engineering
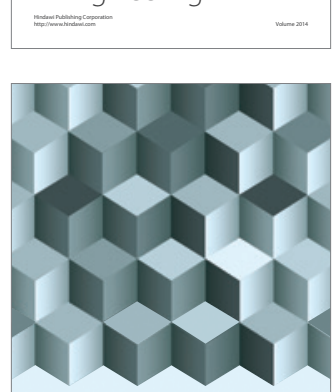

Journal of

Function Spaces
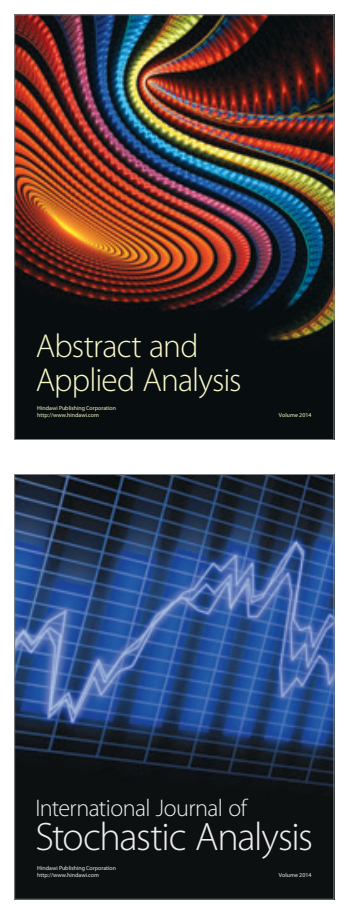

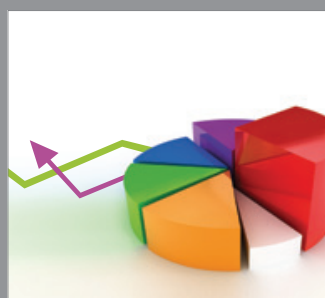

ournal of

Probability and Statistics

Promensencen
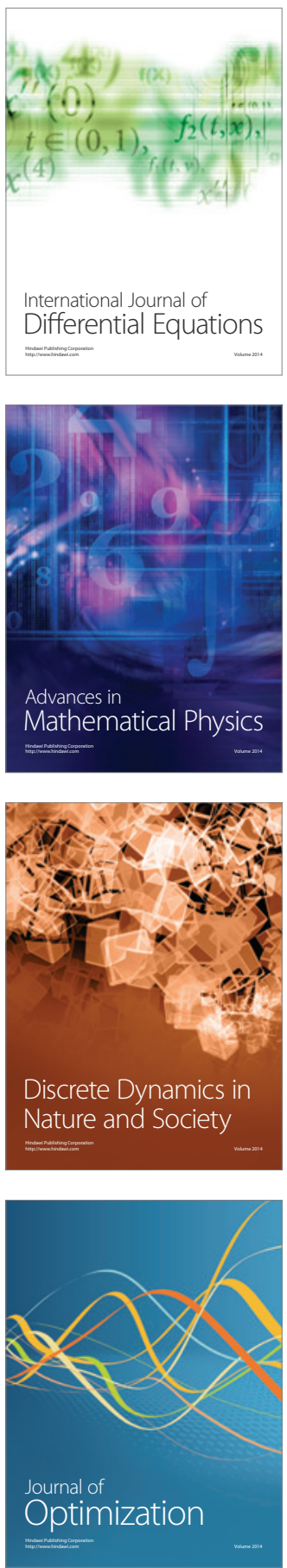